(21.6\%) patients with an active TB diagnosis were not identified by IGRA, TST- $5 \mathrm{~mm}$ and TST-10 $\mathrm{mm}$ respectively. The difference between IGRA and TST was only was only statistically significant between IGRA and TST-10 mm $(p=0.021)$. Agreement between IGRA and TST-5 mm was $\mathrm{k}=0.402$ $(\mathrm{p}<0.001)$ with a concordance rate of $83.5 \%$ and between IGRA and TST-10 mm was $k=0.351(\mathrm{p}<0.001)$ with a concordance rate of $79.5 \%$. Combine sensitivity of IGRA plus TST-5 $\mathrm{mm}$ and IGRA plus TST-10 $\mathrm{mm}$ was $91.7 \%$ and 90.6\%, respectively.

Conclusion IGRA tests showed a high sensitivity, however lower than the TST with a $5 \mathrm{~mm}$ cutoff. The level of agreement between IGRA and TST with either cut-offs was poor, with $16.5 \%$ of the patients showing different outcomes between IGRA and TST-5 mm and $20.5 \%$ between IGRA and TST-10 mm. This significant increase in sensitivity when results from both tests were combined suggests that the use of the two tests together could promote the identification of more cases of infection than if used separately and in substitution of one another. This could be especially important in countries were latent infection is the primary source of $\mathrm{TB}$ cases.

\section{P52 IMPACT OF TWO TYPES OF PHYSICAL ACTIVITY UNDER RISK ENVIRONMENTAL CONDITIONS ON KIDNEY FUNCTION}

${ }^{1}$ UP Santos*, ${ }^{2}$ DMT Zanetta, ${ }^{1}$ MR Leite, ${ }^{3}$ A Cardoso, ${ }^{4}$ EA Burdmann. ${ }^{1}$ Pulmonary Division, Heart Institute-Faculdade de Medicina da Universidade de Sao Paulo, Sao Paulo, Brazil; ${ }^{2}$ Epidemiology, Faculdade de Saude Publica, Universidade de Sao Paulo, Sao Paulo, Brazil; ${ }^{3}$ Universidade Nove de Julho, Sao Paulo, Brazil; ${ }^{4}$ Nephrology Division, Faculdade de Medicina da Universidade de Sao Paulo, Sao Paulo, Brazil

\subsection{6/jech-2019-SSMabstracts.203}

Background Performing strenuous physical activity in a heat environment with high air pollution has been associated to kidney injury in healthy individuals. The aim of this study was to evaluate the acute effects on the kidney of manual harvesting of sugarcane and supervised streets running exercise.

Methods We evaluated 49 male sugarcane workers, three months after harvesting beginning, before and after a daily work shift, and 39 male Brazilian army recruits, six months after performing street running five days a week, before and after a $7.5 \mathrm{~km}(45 \mathrm{~min})$ street running. Urine and blood samples were assessed for inflammatory markers, kidney biomarkers and renal function. Glomerular filtration rate (GFR) was estimated by CKD-EPI equation. Particulate matter $\left(\mathrm{PM}_{2.5}\right)$ and environmental temperature at sugarcane field during working days, and at street circuits during races, were monitored. Continuous variables are described by mean $\pm S D$ or median(IQR).The differences between post and pre values are presented with their 95\% confidence interval. Analyses were performed with SPSS (v21) software.

Results $\mathrm{PM}_{2.5}$ concentration and temperature were higher in sugarcane field: $101.0 \mu \mathrm{g} / \mathrm{m}^{3}$ (IQR: $31.0-139.5$ ) and $29.7^{\circ} \mathrm{C}$ (24.1-34.0) compared to urban environment: $62.0 \mu \mathrm{g} / \mathrm{m}^{3}$ $(37.5-103.0)$ and $22.2^{\circ} \mathrm{C}(20.9-23.5)$. Sugarcane cutters were older $(41.3 \pm 10.6 \times 19.1 \pm 1.0$ years). There was significant and similar reduction of GFR in harvesters and recruits:-15.0 [95\%CI:-18.3;-11.7] $\mathrm{mL} / \mathrm{min} / 1.73 \mathrm{~m}^{2}$ after shift work, and -14.1 $[-18.1 ;-10.1]$ post-run, respectively; similar increase in serum creatinine $(0.17[0.13 ; 0.21]$ and $0.13[0.10 ; 0.16] \mathrm{mg} / \mathrm{dL})$ and in urinary KIM-1 (119.6[-1.5;240.6] and 427.1[168.7;685.5]pg/
$\mathrm{mL})$. There was increase in serum NGAL $(8.9[2.5 ; 15.2] \mathrm{ng} / \mathrm{mL}$ after shift work and $2.7[-18.5 ; 23.9]$ post-run) and in blood monocytes $\left(42.4[2.0 ; 82.7] / \mathrm{mm}^{3}\right.$ after shift work and $-52.6[-$ $120.7 ; 15.4]$ post-run) observed only in sugarcane cutters, and blood neutrophils increase in both groups, which was higher in cutters: $1846[1349 ; 2343]$ vs $400[150 ; 649] / \mathrm{mm}^{3}$ in recruits.

Conclusion Burned sugarcane harvesting and street running were associated with acute decline in kidney function, increase in biomarkers of renal injury and systemic inflammation, with more marked changes in sugarcane workers. These alterations are likely associated with the extraneous physical work, heat stress, air pollution and dehydration. The two groups evaluated showed differences that make it difficult to compare them. However, we were able to show the impact that physical activity in adverse conditions had on the studied outcomes. The effects of a daily repetitive kidney stress and inflammation are unknown, but may evolve to chronic disease in vulnerable individuals. Measures should be taken to improve the working conditions of sugarcane cutters, including cessation of burning of sugarcane, establishment of breaks and better hydration at work.

\section{P53 MATERNAL EDUCATION AND LANGUAGE DEVELOPMENT AT 2 YEARS CORRECTED AGE IN CHILDREN BORN VERY PRETERM: RESULTS FROM A EUROPEAN POPULATION-BASED COHORT STUDY}

${ }^{1}$ MS Sentenac*, 1,2 MLC Charkaluk, ${ }^{3}$ SJ Johnson, ${ }^{1} J Z$ Zeitlin. ${ }^{1}$ Inserm UMR 1153, Center for Epidemiology and Statistics Sorbonne Paris Cité, Paris, France; ${ }^{2}$ Service de néonatologie, Hôpital Saint Vincent de Paul, Groupement des Hôpitaux de I'Institut Catholique Lillois, Lille, France; ${ }^{3}$ Department of Health Sciences, George Davies Centre, University of Leicester, Leicester, UK

\subsection{6/jech-2019-SSMabstracts.204}

Background In the general population, children from socioeconomically disadvantaged families face higher risks of developmental language delay (DLD). Less research exists on very preterm (VPT) children and results have been contradictory, which may reflect a lesser impact of socioeconomic factors when perinatal risks for delayed development are high. Our objective was to investigate the association of maternal education with DLD at 2 years of age by degree of perinatal risk.

Methods Data come from the Effective Perinatal Intensive Care in Europe (EPICE) cohort, a population-based, prospective cohort study of children born $<32$ weeks' gestational age (GA) in 2011/2012. Perinatal data were abstracted from medical records and follow-up was conducted using parental questionnaires at 2 years corrected age. Six countries (Belgium, Estonia, France, Italy, Netherlands, UK) used a validated short form MacArthur Developmental Communicative Inventories Checklist (4666 children at inclusion 2990 (64\%) followed up); DLD was assessed using 2 outcomes: not yet combining words; expressive vocabulary $<10$ th percentile. Families speaking only other languages at home were excluded. Modified Poisson regression models were used to estimate relative risks (RRs) for DLD for maternal education overall and by perinatal risk (low, moderate, high), classified using GA, small for gestational age (SGA) and severe neonatal morbidities. All analyses were performed using Stata version 15 .

Results 2643 VPT children (mean GA 28.8 weeks) were assessed at a median 24 months corrected age. 25.3\% were not combining words and almost $40 \%$ were $<10$ th percentile for expressive vocabulary. Among children with low perinatal 